\title{
Intervenciones internacionales ciudadanas y la resistencia no-violenta en los Territorios Palestinos ${ }^{2}$
}

\author{
International Citizenship Intervention and \\ the No-violent Resistance in the Palestinian Territories
}

\begin{abstract}
Resumen
Este artículo de investigación realiza una aproximación al fenómeno de la resistencia civil en los Territorios Palestinos contra la ocupación y a los efectos del apoyo noviolento que recibe desde sectores de la sociedad civil global. La resistencia civil tiene, en la historia del movimiento nacional palestino, un papel mayoritariamente subordinado a la lucha armada. Sin embargo, esto ha cambiado en los últimos años donde la lucha no-violenta predomina como estrategia de resistencia. En este contexto, las intervenciones internacionales ciudadanas están beneficiando a los movimientos palestinos que practican esta resistencia mediante el aumento de su protección y favoreciendo su empoderamiento. Además, están extendiendo la cultura de paz y desafiando el militarismo existente en las sociedades israelí y palestina.
\end{abstract}

Palabras clave: Resistencia civil, transformación de conflictos, Oriente Medio, sociedad civil global.

\begin{abstract}
This research article states an approach to the phenomenon of civil resistance against occupied Palestinian territories and the effects of non-violent support that is provided from sectors of global civil society. The civil resistance has a subordinated role mainly to armed fighting in the history of national Palestinian movement. However, this armed fighting has lately changed where non-violent fight prevails as a strategy of resistance.

In this context, international citizenship interventions support those Palestinian movements that put into practice this resistance through the increase of their protection that helps their empowerment. Moreover, international interventions increase the peace culture and challenge the existing militarism that prevails at Israeli and Palestinian society.
\end{abstract}

Keywords: civil resistance, transformation of conflicts, Middle East, global civil society.

Recibido el 12 de noviembre de 2013 y aprobado el 14 de noviembre de 2013

1 Doctor en Historia Contemporánea. Investigador del Centre for Peace and Reconciliation Studies Coventry University. Correo electrónico: diegochecahidalgo@gmail.com

2 Artículo resultado de la investigación "Intervenciones internacionales noviolentas en los Territorios Palestinos". 


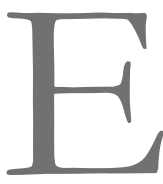

n el contexto de las primaveras árabes, donde las reivindicaciones sociales, protestas ciudadanas y transiciones democráticas han supuesto una sacudida para los regímenes autoritarios del mundo árabe, una parte de la sociedad palestina se encuentra embarcada en un proceso de resistencia civil contra la ocupación israelí de los Territorios Palestinos y sus consecuencias. En esa lucha, los movimientos locales no se encuentran solos, sino que cuentan con el apoyo de sectores de la sociedad civil global que simpatizan con su causa y demandan el respeto de los derechos humanos y el cumplimiento de la legalidad internacional. Y es la actuación de esta sociedad civil global que pone en marcha intervenciones internacionales ciudadanas, la que es objeto de análisis en este artículo.

La historia ha puesto de manifiesto que los movimientos pacifistas y noviolentos tienen una enorme capacidad transformadora frente a las estructuras políticas, económicas, sociales y culturales. En general, los movimientos sociales han orientado esta capacidad transformadora de la noviolencia hacia la construcción de la paz en sociedades más justas y sostenibles, generando hoy en día movimientos protagonizados por ciudadanos en acción, que creen en el respeto de los derechos humanos y en la defensa de sociedades más libres, democráticas, equitativas, sostenibles, solidarias y tolerantes. Un conjunto de ciudadanos, personas normales y corrientes, que conforman una sociedad civil global solidaria que persigue un globalismo de abajo hacia arriba, desde los movimientos de base y más allá de las elites y los tradicionales poderes políticos.

Las intervenciones internacionales noviolentas nacieron y evolucionaron a partir de este sustrato. Son intervenciones ciudadanas desarrolladas para actuar en el ámbito de la resolución de conflictos en el escenario internacional más allá de los dictados y de los intereses de los respectivos estados y gobiernos de sus protagonistas. Son herramientas creadas por la sociedad civil, desde el pacifismo, para promover la transformación de conflictos y la construcción de una paz positiva en los sentidos apuntados por John Paul Lederach (1997) y Johan Galtung (1969). Y en el caso de Palestina toman la forma de activistas que protegen a manifestantes no-violentos de la represión de las fuerzas de seguridad, facilitan el empoderamiento de comunidades vulnerables amenazadas por el desplazamiento forzoso, defienden a pastores y campesinos intimidados por colonos o acompañan a niños que sufren acoso en su desplazamiento diario al colegio, por citar solamente algunos ejemplos.

En este artículo realizaremos una breve aproximación al fenómeno de la resistencia civil en los Territorios Palestinos contra la ocupación israelí y a los efectos del apoyo internacional noviolento que recibe desde distintos sectores de la sociedad civil global, apoyándonos en un trabajo de campo realizado en dos periodos distintos entre los años 2011 y 2013 que nos permitió recoger información a partir de fuentes primarias (entrevistas semi-estructuradas a informantes clave y observación participante en acciones directas). Primero introduciremos algunos apuntes básicos sobre las intervenciones internacionales ciudadanas. Después repasaremos brevemente la historia del movimiento nacional palestino y su lucha contra la ocupación, centrándonos especialmente en los aspectos relacionados con la resistencia civil. A continuación examinaremos las distintas intervenciones internacionales noviolentas que se desarrollan en los Territorios Palestinos, destacando los resultados que han logrado y señalando sus principales limitaciones. Finalmente presentaremos algunas conclusiones en torno a las potencialidades que este fenómeno encierra para los movimientos noviolentos que persiguen transformaciones sociales y políticas en Oriente Medio.

\section{Las intervenciones internacionales ciudadanas}

Cuando hablamos de intervenciones internacionales ciudadanas nos referimos a un fenómeno protagonizado por civiles que se organizan de manera transnacional para transformar conflictos con altos niveles de violencia que acontecen en diver- 
sas partes del mundo, afectando a organizaciones y grupos vulnerables. Son actuaciones desarrolladas para contribuir a la construcción de una paz duradera y sostenible mediante la utilización de estrategias de acción noviolenta (Checa Hidalgo, 2011). Este fenómeno cuenta con una silenciosa trayectoria en el tiempo, aunque ha tenido una creciente popularización en las últimas décadas, lo que se ha manifestado en un significativo aumento del número y del tamaño de las iniciativas que se han puesto en marcha para desarrollar diferentes actividades en el campo de la gestión de conflictos en el escenario internacional.

El origen de este tipo de intervenciones podemos vincularlo a dos desarrollos que ocurrieron a comienzos del siglo XX. El primero es la propuesta presentada por William James para utilizar alternativas civiles noviolentas a fin de gestionar los conflictos entre diferentes estados. James, tras constatar las consecuencias tan negativas que la guerra y el militarismo tenían para la sociedad, promovió el desarrollo de un servicio civil de paz como una herramienta para la resolución de conflictos que sustituyese a los ejércitos (James, 1910). El segundo está unido a la figura de Gandhi, quien dio continuidad a esta idea al proponer la creación de un ejército de paz, o Shanti Sena, equivalente al servicio militar tradicional (Cortright, 2008, p. 310), que comprendiese actividades que pueden considerarse como prevención de la violencia, peacekeeping, peacemaking y peacebuilding (Weber, 1996). Pero, más allá de dichas formulaciones, las campañas noviolentas de Gandhi en Sudáfrica y, sobre todo, en la India, mostraron la capacidad que tenían dichas campañas para alcanzar objetivos políticos en conflictos asimétricos con un notable diferencial de poder entre las partes implicadas en los mismos. Gandhi mostró que la noviolencia podía ser una herramienta poderosa para el movimiento pacifista.

Los intentos por organizar intervenciones internacionales ciudadanas que frenasen la utilización de los ejércitos y de la guerra para resolver las disputas entre los estados continuaron después de la Primera Guerra Mundial, aunque no llegaron a tomar formas concretas. La Segunda Guerra
Mundial hizo que parte del movimiento pacifista cuestionase la utilización de la metodología gandhiana de manera efectiva en todos los escenarios, por lo que empezó a calar la idea de llevar a cabo intervenciones a gran escala de ejércitos pacíficos por intervenciones realizadas a menor escala, utilizando el marco legal creado por la nueva configuración del sistema internacional tras la guerra. Así empezaron a diseñarse y a implementarse estas nuevas intervenciones internacionales a menor escala para la transformación noviolenta de conflictos, aunque sus resultados hasta finales de la Guerra Fría fueron muy desiguales.

El despegue definitivo de las intervenciones ciudadanas se produce a comienzos de la década de 1980, cuando el estallido de varias guerras en América Central atrajo el foco del movimiento pacifista a esta área geográfica. De esta manera, construidas sobre el conocimiento acumulado desde comienzos del siglo XX, iniciaron su andadura una serie de organizaciones de base cuyo objetivo era trabajar por la transformación de los conflictos sobre los que actuaban, mediante el despliegue de equipos de paz sobre el terreno. Así nacieron Peace Brigades International (1981), Witness for Peace (1981), Christian Peacemaker Teams (1986) y Pastors for Peace (1988), entidades que posteriormente trasladarían su experiencia a otros escenarios internacionales donde han continuado desarrollando sus actividades. De este modo, estas organizaciones sirvieron como germen para la materialización de esa vieja aspiración del movimiento pacifista que abogaba por la viabilidad de la puesta en marcha de un servicio civil noviolento de carácter internacional para la intervención en conflictos (Checa Hidalgo, 2012, p. 29).

Esa nueva fase de desarrollo de las intervenciones internacionales ciudadanas tuvo su continuidad tras el fin de la Guerra Fría gracias a los cambios acontecidos en la sociedad internacional, a la experiencia acumulada por las organizaciones que las practicaban y al efecto multiplicador que generaban los resultados positivos de su trabajo. Todo ello hizo que el número y el volumen de estas iniciativas creciese y el fenómeno se fuese expandiendo. Los conflictos en Europa del Este 
durante los años noventa les otorgaron diferentes escenarios para su actuación y fueron el desencadenante de que, debido a una mayor movilización del movimiento pacifista internacional, nacieran nuevos grupos y organizaciones que desarrollaban estrategias noviolentas para la transformación de conflictos. A partir de la década de 1990 también se han producido intervenciones internacionales noviolentas en Oriente Medio, América Latina y, en menor medida, tanto en Oceanía como en Asia. ${ }^{3}$

En resumen, podemos decir que el fenómeno de las intervenciones internacionales ciudadanas noviolentas y el aumento de su protagonismo en el campo de la gestión de conflictos se ha potenciado gracias tanto a los cambios acontecidos en la sociedad internacional como al sustrato generado por el movimiento pacifista a lo largo del siglo XX. De esta forma, en la actualidad se presentan como unas herramientas eficaces para la prevención de la violencia y la transformación de conflictos en múltiples escenarios. En este artículo nos centraremos en analizar su trabajo en el caso del conflicto israelí/palestino.

\section{Lucha y resistencia palestina contra la ocupación}

La sociedad palestina tiene una prolongada trayectoria de resistencia contra la ocupación de su territorio que ha adoptado formas de lucha tanto violentas como no-violentas a lo largo de la historia. Esta resistencia se manifestó primero en contra del Imperio Otomano y luego contra el Mandato Británico y la emigración judía a este territorio. Más tarde, la culminación del proyecto sionista con la creación del Estado de Israel en 1948 y su posterior expansión hacia los territorios de Cisjordania y Gaza en 1967, convirtieron a la ocupación israelí en el principal objetivo de la resistencia palestina. En cada etapa de esta resistencia contra las ocupaciones, tal y como señala el profesor Andrew Rigby, la mayoría de la población resistió principalmente mediante medios no-violentos. Sin embargo, con excepción del periodo de la primera intifada (1987-1991), esos modos de resistencia no-violentos fueron subordinados a otros medios de lucha caracterizados por la retórica y la práctica de la violencia (Rigby, 2010).

En nuestra opinión, el conflicto palestino-israelí hunde sus raíces en la colisión, a comienzos del siglo XXI, de dos proyectos nacionales: el sionista y el árabe-palestino. El proyecto sionista se conformó primero, surgiendo a finales del siglo XIX e impulsado intelectualmente por Theodor Herzl, con la intención de guiar al movimiento nacional judío hacia la colonización de Palestina (Pappe, 2004, p. 36). Aunque hacia finales de la primera década del siglo XX el fenómeno sionista contaba con menos de 50.000 personas en Palestina, el antagonismo con la población local era manifiesto y se expresaba a través de diferentes formas de resistencia, especialmente contra la venta de tierra a las organizaciones sionistas. Como resultado de la irrupción del fenómeno sionista en la región, la población nativa desarrolló sus propios sentimientos nacionales, proceso que se intensificó como resultado de la presencia colonial británica. Los acuerdos de Sykes-Picot y la Declaración Balfour convirtieron en papel mojado las promesas sobre la independencia que Gran Bretaña realizó a los árabes durante la Primera Guerra Mundial, ${ }^{4}$ e impulsaron la orga-

3 Para completar este breve repaso histórico a las intervenciones internacionales noviolentas acontecidas desde mediados del siglo XX y conocer el desarrollo y los resultados que han obtenido, pueden consultarse los trabajos de los profesores MoserPuangsuwan (2000) y Weber (1993).

4 Los acuerdos de Sykes-Picot fueron firmados en 1916 por Francia y Gran Bretaña para establecer sus áreas de influencia sobre los territorios del Imperio Otomano en Oriente Medio. Estos acuerdos guiaron la configuración de la región tras la derrota otomana gracias al respaldo obtenido de la Liga de las Naciones que autorizó el Mandato Británico sobre Palestina en 1922. En ese mismo periodo se produjo el segundo hecho, la llamada Declaración Balfour que llevó al gobierno británico a apoyar la creación de un hogar nacional judío en Palestina, en 1917 (Pappe, 2004). 
nización de seis congresos árabes palestinos entre 1919 y 1923, así como protestas y manifestaciones en contra de la Declaración y en favor de la creación de un gran Estado árabe en Oriente Medio, impulsando el sentimiento nacional palestino (King, 2007).

Durante las décadas previas a la creación del Estado de Israel, la sociedad palestina mostró su resistencia a la ocupación, especialmente contra las medidas que facilitaban el desarrollo del proyecto sionista (Rigby, 2010). También la resistencia contra el Mandato Británico tuvo un importante componente no-violento. Inicialmente incluyó múltiples métodos de protesta y persuasión como declaraciones, peticiones, asambleas y marchas, entre otros. También fueron utilizados métodos de no-cooperación como la no participación en el sistema político, la realización de huelgas, el boicot a productos judíos y británicos, la insumisión fiscal $\mathrm{y}$ acciones de desobediencia civil. Posteriormente, la resistencia civil palestina se extendió e intensificó durante la década de 1930, mientras la inmigración judía se incrementó exponencialmente. Así, la sociedad palestina llevó también a cabo actos de no-cooperación como la suspensión de actividades sociales, la retirada de las instituciones, la ruptura de la colaboración con los administradores británicos, la renuncia a puestos oficiales y, a menudo, la desobediencia civil (King, 2007, pp. 33-43). Aunque durante este periodo la resistencia civil fue mayoritaria, la violencia también fue un recurso de lucha utilizado por el movimiento nacional palestino (Rigby, 2010, pp. 23-30).

Gran Bretaña decidió en 1947 poner fin al Mandato y trasladar la responsabilidad sobre Palestina a la recién creada Organización de Naciones Unidas (ONU), quien optó por dividir el territorio para crear dos estados que respondiesen a las demandas de los proyectos nacionales árabe-palestino y sionista (Pappe, 2004, pp.
123-129). La acogida de las poblaciones locales fue diametralmente distinta y Palestina se encaminó hacia la guerra civil. Cuando el 15 de mayo de 1948 Israel se proclamó Estado independiente y los ejércitos de la Liga Árabe entraron en escena, las atrocidades se sucedían ya contra las poblaciones árabes y las judías por parte de los grupos armados palestinos y sionistas (Rigby, 2010, pp. 31-32). El resultado de la primera guerra árabe-israelí fue la derrota de los ejércitos árabes, la anexión israelí de territorios que originalmente habían sido destinados al Estado palestino por la ONU y el desplazamiento y la opresión de la población palestina bajo la dominación de Israel, Jordania y Egipto. En este contexto, las iniciativas no-violentas tuvieron un impacto muy limitado y probaron ser ineficaces, salvo casos puntuales. ${ }^{5}$

Tras el desastre de 1947-48 y hasta la creación de la Organización para la Liberación de Palestina (OLP) en 1964, casi no hubo manifestaciones públicas significativas del nacionalismo palestino de ningún tipo. Las consecuencias de la guerra hicieron que las prioridades de los palestinos se centraran en la pura supervivencia, pero esta ausencia también se explica por la fe en el triunfo del panarabismo (Rigby, 2010, pp. 41-42). Este periodo vio la emergencia de otro tipo de resistencia encarnada en la firme resolución de los palestinos de permanecer en la tierra, resistir la expulsión y perseverar en la vida diaria a pesar de la ocupación (Norman, 2010, p. 21).

La ocupación de Cisjordania y Gaza en 1967 por Israel y la creación de la OLP supusieron el fin de la apuesta por el panarabismo. Poco a poco la OLP aglutinó las diferentes facciones del movimiento nacional palestino y emprendió una resistencia armada en sintonía con las guerras de liberación que acompañaban muchos procesos descolonizadores en esos años. Mientras el reclu-

5 Uno de esos casos fue el de Battir, un pequeño pueblo palestino que se salvó de la destrucción durante y después de la guerra gracias a una estrategia creativa de resistencia civil basada en un fuerte liderazgo, la solidaridad y el compromiso en torno a una causa y la ausencia de contra movimientos (Rigby, 2010, pp. 35-40). Su experiencia contrasta con las más de 500 aldeas y pueblos palestinos que fueron destruidos durante este periodo (Pappe, 2006). 
tamiento para la resistencia armada se producía en los campos de refugiados fuera de los territorios ocupados, en el interior se producía un proceso de organización civil y política guiado inicialmente por el partido comunista que abogaba por la participación popular y por la utilización de métodos de resistencia no-violenta, al que siguieron el resto de facciones tras la derrota árabe de 1973 (King, 2007). Esta "organización popular", durante las décadas de 1970 y 1980, consistió en la construcción de amplias redes de organizaciones sociales de base, creadas para proporcionar infraestructura política, social y cultural bajo la ocupación. Era un esfuerzo de resistencia civil desarrollado por asociaciones de mujeres, estudiantes o profesionales, asociaciones sociales y culturales que se convirtieron en redes de movilización política, proporcionaron servicios a la comunidad y mantuvieron la identidad palestina (King, 2007). Durante este periodo, y a pesar de la ocupación militar, la incipiente sociedad civil palestina se volcó en un esfuerzo constructivo que extendió la acción colectiva, estableciendo instituciones alternativas, desarrollando procedimientos democráticos y adquiriendo estrategias de acción civil que llevarían a la creación de la primera intifada (Norman, 2010, p. 23).

La primera intifada (1987-1991) fue un levantamiento popular acontecido en los territorios ocupados palestinos para luchar por sus derechos y contra la ocupación israelí. La resistencia se convirtió en un fenómeno generalizado que implicó a casi todas las capas de la sociedad palestina y fue mayoritariamente no-violenta, aunque en ocasiones contempló el lanzamiento de piedras con la intención de infringir daño a las fuerzas ocupantes. La intifada utilizó una múltiple combinación de métodos de lucha, recurriendo a la resistencia simbólica vistiendo ropas tradicionales, a la nocooperación económica participando en huelgas, a la no-cooperación política abandonando sus puestos en la administración israelí, a la ocupación del espacio público para enfrentarse directamente al ejército opresor, al desarrollo de redes de apoyo para ayudar a los manifestantes y a los huelguistas, a la creación de nuevos espacios productivos como el que supuso la extensión de los cultivos a los jardines y al establecimiento de un sistema educativo clandestino (King, 2007).

Pero la intifada, a pesar de su componente nacionalista y su aspiración de poner fin a la ocupación israelí, no culminó en la formación de un Estado palestino, sino en la firma de los Acuerdos de Paz de Oslo (en 1993 y 1995) para la creación de una región semiautónoma en parte de los territorios ocupados y gobernada por la Autoridad Palestina (AP). Llegados a ese punto, parecía que el proceso de Oslo culminaría en la creación del Estado palestino a partir de la extensión de la autonomía y que la AP se transformaría en el gobierno nacional de Palestina. Sin embargo, el proceso de Oslo solo mantuvo el status quo y la extensión de la opresión política y económica de Cisjordania y Gaza, donde los asentamientos israelíes continuaron creciendo. Esta situación desilusionó a la sociedad palestina y la frustración y la rabia se extendieron, dando origen a la segunda intifada en el año 2000. La segunda intifada continuó hasta el año 2005 y consistió en el predominio de una resistencia violenta que incluyó enfrentamientos armados y ataques terroristas. Esta escalada de la violencia en el conflicto también aumentó la violencia de la represión de las fuerzas de seguridad israelíes. De este modo, las actividades de resistencia civil fueron más limitadas, implicaron a una menor proporción de la población y fueron subordinadas a las estrategias militares. Este dominio de la vía militar alejó también la simpatía de la opinión pública internacional del bando palestino, cuyo evidente sufrimiento ante la represión israelí de la primera intifada había generado una amplia solidaridad internacional.

Durante la segunda intifada comenzaron a configurarse algunos procesos de resistencia no armada que perdurarían en el tiempo y que, poco a poco, se fueron convirtiendo en los elementos más visibles de la resistencia palestina contra la ocupación. De esta manera, la vía no-violenta ha llegado a suponer, en nuestra opinión, la principal estrategia de lucha en el movimiento nacional palestino en la era de la primaveras árabes, ya que aunque una gran parte de la población palestina sigue defendiendo el derecho a utilizar cualquier 
medio para lograr el fin de la ocupación, el apoyo a la lucha armada está alcanzando niveles mínimos (Seitz, 2011, pp. 11-12). Nuestro trabajo de campo nos ha permitido identificar cuatro grandes áreas de lucha no-violenta contra la ocupación israelí de los Territorios Palestinos construidos sobre la tradición de la resistencia civil palestina: la lucha contra la desposesión de la tierra; contra la colonización israelí; contra el bloqueo y la privación de libertad de movimiento; y contra la privación de libertades personales. A continuación repasaremos brevemente en qué consisten cada una de ellas.

La lucha contra la desposesión de la tierra se produce en múltiples frentes a lo largo de los Territorios Palestinos, pero el más visible de ellos es la lucha contra el muro. La segunda intifada impulsó la decisión israelí de construir una barrera física entre Cisjordania e Israel, un muro que comenzó a construirse en 2002. Mientras el gobierno israelí justificaba el muro en términos de seguridad para impedir el acceso de terroristas a Israel, muchos observadores señalaron el interés israelí en expropiar territorio palestino, puesto que su diseño no seguía la demarcación de la Línea Verde. ${ }^{6}$ El muro provocó una ola de resistencia entre aquellos más directamente afectados a los que se les impedía el acceso a sus tierras de cultivo o de pastoreo y se amenazaba su supervivencia. Así que los habitantes de los pueblos y aldeas por los que avanzó la construcción del muro se organizaron en movimientos de base, coordinados a través de los comités populares que incluían a cualquier habitante independientemente de su afiliación política. El proceso comenzó en Budrous en 2003 y se extendió por los Territorios Palestinos hasta llegar a formarse más de 50 comités, ampliando la resistencia a otras formas de desposesión de la tierra que la ocupación israelí llevaba a cabo. El trabajo de los comités consiste en coordinar las acciones directas realizadas contra la desposesión de la tierra que toman la for- ma de manifestaciones, marchas y otros tipos de protesta no armada. Además apoyan las acciones legales llevadas a cabo en los tribunales israelíes dirigidas a la modificación del trazado del muro, a evitar la expropiación de propiedades o a la liberación de los activistas detenidos (Broning, 2011). Esta resistencia ha tenido en ocasiones notorios éxitos, como cuando logró modificar el trazado del muro en Budrous y Bi'lin, o cuando evitó el desplazamiento de la población palestina que vive en las colinas del sur de Hebrón. Junto a esos éxitos también debemos mencionar las limitaciones del alcance de esta resistencia que se hacen evidentes tan pronto se visualiza el trazado actual del muro y el elevado tributo que, en forma de muertos, heridos, detenidos y castigos colectivos generados por las fuerzas de seguridad ocupantes, tiene que pagar la población que la practica.

La lucha contra la colonización tiene su máxima expresión en la campaña Boicot, Desinversiones y Sanciones (BDS). Esta campaña fue iniciada en el año 2004 y, poco después, se convirtió en una iniciativa impulsada por organizaciones de la sociedad civil palestina para pedir a la comunidad internacional que presionase a Israel con boicots, desinversiones y sanciones hasta que se respetase la legalidad internacional (Barghouti, 2006). Este movimiento, que pronto alcanzó un impacto mundial, pretende elevar los costes del mantenimiento de la ocupación para Israel y, para ello, alienta el boicot de consumidores a productos y servicios de empresas israelíes o de empresas con intereses en Israel y las desinversiones en dichas empresas. El boicot se solicita también en ámbitos académicos, culturales y deportivos, con la intención de deslegitimar a los representantes de un Estado que incumple la legislación internacional y viola sistemáticamente los derechos humanos, y a los que se benefician de él, y llama a sancionar al Estado de Israel y a sus representantes de acuerdo al derecho internacional, suspendiendo o cancelando las relaciones de cooperación con el gobierno israe-

6 La Línea Verde es la línea de demarcación establecida en los acuerdos de armisticio firmados en 1949 entre Israel y sus vecinos (Egipto, Jordania, Líbano y Siria) después de la Primera Guerra Árabe-Israelí de 1948. Su trazado establece las fronteras internacionales del Estado de Israel tal y como reconoce la resolución 242 del Consejo de Seguridad de la ONU. 
lí (Broning, 2011). Una muestra de estos efectos son la suspensión de las relaciones militares de Turquía y Noruega con Israel, o las recomendaciones del Parlamento Europeo para imponer un boicot sobre los productos israelíes procedentes de los asentamientos en los territorios ocupados.

La resistencia palestina también se ha centrado en la lucha contra la privación de movimientos y contra el bloqueo que, en particular, sufre la población de la franja de Gaza. ${ }^{7}$ Este bloqueo ha generado un fuerte rechazo y una importante solidaridad internacional que fue canalizada en apoyo de grupos de activistas palestinos e internacionales decididos a desafiar el bloqueo sobre Gaza mediante campañas de resistencia civil y acción directa noviolenta. Así se creó el Free Gaza Movement para intentar romper el bloqueo israelí por mar, distribuir ayuda humanitaria y desplegar observadores internacionales. El movimiento ha organizado convoyes de ayuda a Gaza desde 2008 que han obtenido un éxito desigual, consiguiendo romper el bloqueo en cuatro de las ocho ocasiones en las que lo han intentado (De Jong, 2012, p. 201). Las acciones de estos convoyes y las respuestas israelíes, sobre todo con el ataque a la 'Flotilla de la Libertad', aumentó la presión de la comunidad internacional sobre Israel para que suavizara el bloqueo, cosa que acabó sucediendo. Este hecho mostró a los líderes de Hamas que por medio de la lucha no-violenta se podían conseguir resultados que no había logrado la lucha armada y éstos empezaron a valorarla como una forma de resistencia factible (Broning, 2011).

La cuarta de las áreas prioritarias para la resistencia civil palestina ha sido la lucha contra la privación de libertades personales y a favor de la liberación de los presos políticos. La problemática de los presos políticos tiene gran calado en la sociedad palestina. ${ }^{8}$ Los presos personalizan la resistencia del movimiento nacional palestino y su sacrificio inspira un profundo respeto en la sociedad palestina. En las cárceles israelíes, los presos se han organizado y han desarrollado acciones y campañas de desobediencia civil y resistencia no-violenta. En estas acciones, las huelgas de hambre han sido una táctica recurrente desde la primera intifada para mejorar sus condiciones de vida en prisión (Qumsiyeh, 2011, p. 195). Fuera de ellas se han organizado numerosas campañas en apoyo de sus peticiones ante las instituciones israelíes y ante los representantes de la comunidad internacional. Normalmente, sus familias y amplios sectores de la sociedad civil secundan las acciones de los prisioneros con manifestaciones y protestas y, en ocasiones, se han unido a las huelgas de hambre. Los éxitos de estas campañas son pequeños, pero han contribuido a la mejora de las condiciones de vida en prisión para los presos políticos palestinos a partir de medidas como poder vestir ropas civiles, tener acceso a las noticias, conseguir derechos de visita razonables y mejorar su acceso a los servicios sanitarios. Además, debido al alto porcentaje de palestinos que han pasado por cárceles, éstas han ocupado un lugar importante en el proceso de socialización de la resistencia civil, ya que muchos activistas comenzaron a practicar la resistencia no-violenta en prisión porque era el único medio de lucha a su alcance allí, lo que $a$ posteriori se convirtió en un importante aprendizaje (Norman, 2010, pp. 65-68).

7 Durante la segunda intifada Israel se retiró de Gaza, aunque se reservó el control de sus fronteras y del espacio aéreo y naval. Cuando Hamas ganó las elecciones legislativas palestinas de 2006 y, poco después, se hizo con el poder en Gaza, la comunidad internacional le impuso sanciones económicas e Israel y Egipto cerraron sus fronteras con ella debido a su actividad terrorista y a su no reconocimiento del Estado de Israel. Desde entonces, su 1.500.000 de habitantes ve muy limitada su conexión con el exterior y su economía ha quedado destruida, con el consiguiente impacto humanitario.

8 Desde 1967 más de 800.000 palestinos han sido detenidos por Israel, lo que supone el 20\% del total de la población en los Territorios Palestinos y, significa, que cerca del $40 \%$ de los hombres que viven allí han estado en prisiones israelíes (Addameer, 2012). En las cárceles, los prisioneros sufren la detención sin cargos o juicio, reclusión en condiciones de aislamiento y el rechazo de las visitas de familiares desde Gaza. Existen informes que describen el uso de la tortura en ellas y la negación de tratamiento médico adecuado (Amnesty International, 2013). 
Este breve repaso a la trayectoria del movimiento nacional palestino nos muestra cómo a lo largo de su historia ha utilizado tanto la lucha armada como la lucha no-violenta en su resistencia contra la ocupación. Nuestra investigación revela que, después de la segunda intifada, la resistencia civil se ha manifestado como el medio de lucha predominante aunque no exclusivo. Sin embargo, si valoramos la capacidad para ser efectiva de la resistencia civil palestina tras la segunda intifada utilizando los indicadores que ofrecen el profesor Andrew Rigby o las investigadoras Maria Stephan y Erica Chenoweth (Rigby, 2010; Chenoweth \& Stephan, 2011), debemos señalar que el movimiento nacional palestino aun está lejos de resultar efectivo. Es cierto que ha desarrollado estrategias creativas que les permiten maniobrar en medio de la represión y que ha conseguido atraer una participación diversa que supera las fracturas políticas de la sociedad palestina, pero aún no ha alcanzado una masa crítica significativa, ni ha provocado cambios relevantes en las políticas israelíes ni en sus pilares de apoyo. Para su sostenibilidad cuenta a su favor con el amplio reconocimiento internacional de la legitimidad de sus reivindicaciones y con una creciente capacidad para movilizar apoyos externos, especialmente en la sociedad civil global. Es en este punto donde las intervenciones internacionales ciudadanas deben jugar un papel fundamental.

\section{El apoyo internacional noviolento a la resistencia civil palestina}

Como hemos mencionado en el apartado anterior, la primera intifada tuvo como uno de sus principales resultados el cambio que la percepción que la opinión pública y parte de la sociedad civil internacional tenían con respecto al conflicto israelí/palestino, lo que llevó a modificar la naturaleza de las intervenciones internacionales ciu- dadanas en la zona. Hasta la primera intifada, las intervenciones internacionales ciudadanas consistieron principalmente en actos de solidaridad con la sociedad palestina que no implicaban la presencia de activistas en la zona de conflicto, lo que ha seguido siendo la forma de intervención más común. Sin embargo, junto a ellas, desde 1987 existen un mayor número de intervenciones ciudadanas noviolentas que precisan de la presencia de activistas internacionales sobre el terreno. ${ }^{9}$

La actividad del Palestinian Center for Rapprochement between People (PCR) ilustra este cambio. El PCR comenzó en 1989 a movilizar el apoyo de activistas y organizaciones de la sociedad civil internacional para fortalecer la resistencia no-violenta palestina contra la ocupación. Un ejemplo de ello fue su impulso a la creación de un campamento de la paz, que contó con presencia permanente de activistas locales e internacionales durante cuatro meses, para protestar contra la construcción del asentamiento de Har Homa, cerca de Jerusalén Este (Qumsiyeh, 2011, p. 171). A partir de los inicios de la segunda intifa$d a$, su trabajo facilitó la formación del International Solidarity Movement. Es a partir de ese momento, en el contexto de la segunda intifada, cuando se puede apreciar un crecimiento de las iniciativas ciudadanas que van a identificar situaciones donde intervenir de manera noviolenta para prevenir la violencia y apoyar procesos de empoderamiento palestinos en torno a la resistencia civil.

\section{Intervenciones internacionales ciudadanas en el conflicto Israelí/Palestino}

Nuestra investigación sobre el apoyo internacional noviolento a la resistencia civil palestina se ha centrado en aquellas intervenciones ciudadanas de carácter transnacional que se realizan on-site, es decir, mediante el despliegue de activis-

9 En esa línea, el profesor Rigby identifica cuatro tipos de intervenciones ciudadanas llevadas a cabo por la sociedad civil internacional en los Territorios Ocupados de acuerdo con los objetivos de la acción: protesta; apoyo y solidaridad; ayuda humanitaria; y resolución del conflictos (Rigby, 1995). En su trabajo se pueden encontrar múltiples ejemplos de dichas desde 1987 hasta 1995. 
tas sobre el terreno donde se produce el conflicto (Rigby, 1995), y que se desarrollan mediante proyectos con un marcado enfoque a largo plazo que persiguen la prevención de la violencia, la transformación del conflicto y la construcción de paz. De acuerdo a estos parámetros, hemos podido identificar seis organizaciones que llevaban a cabo estas iniciativas en el periodo en el que se ha realizado nuestra investigación (2011-2013): Christian Peacemaker Teams (CPT); International Solidarity Movement (ISM); Eccumenical Accompaniment Programme in Palestine and Israel (EAPPI); International Women's Peace Service (IWPS); Operazione Colomba (OC); y el Instituto Internacional para la Noviolencia Activa (NOVACT).

La iniciativa que tiene la trayectoria más longeva en la región es Chrisitan Peacemaker Teams que ha estado trabajando de manera ininterrumpida desde 1995. CPT es una organización de base que surgió en 1986 a partir de las tradiciones de las iglesias anabaptistas menonitas que abogaban por la lucha noviolenta para la consecución del cambio social y terminar con la violencia y la injusticia. Desde entonces ha desplegado equipos de paz en áreas de conflicto como Colombia, Irak, Haití, México, Israel/Palestina e, incluso, en EE.UU. y Canadá. La llegada de CPT a los Territorios Palestinos fue el resultado de las experiencias adquiridas por una serie de delegaciones de la organización que consiguieron establecer relaciones con palestinos e israelíes afectados por la ocupación de Cisjordania por parte de Israel. En 1995, CPT recibió una carta de invitación del alcalde de Hebrón para el establecimiento de un equipo de reducción de violencia en la ciudad que se enfrentaba a un explosivo contexto tras la firma de los Acuerdos de Oslo. ${ }^{10} \mathrm{El}$ proyecto ha trabajado en la ciudad desde entonces, apoyando la resistencia noviolenta de la sociedad palestina contra la ocupación en coordinación con otras organizaciones israelíes e internacionales (Kern, 2000, pp. 188-192). Para ello ha supervisado el tratamiento de la población palestina que cruza los controles militares en el centro histórico y ha intervenido ante violaciones de los derechos humanos cometidas durante las invasiones militares de las tiendas y hogares de los palestinos por las fuerzas de seguridad israelíes. Más allá de la ciudad de Hebrón, CPT ha trabajado en la cercana aldea de At-Tuwani para proteger a la población de los ataques de colonos, especialmente a los niños que son acosados durante su desplazamiento diario a la escuela y a los pastores y agricultores cuando trabajan en el campo.

El International Solidarity Movement es, como ellos mismos se definen, "un movimiento liderado por palestinos comprometidos con la resistencia a la opresión y al despojo de la población palestina, utilizando los principios y métodos de acción directa no-violentos", ${ }_{11}$ que fue fundado en el verano de 2001, en el contexto de la segunda intifada, para apoyar y fortalecer la resistencia civil palestina y pronto se convirtió en la cara más visible del activismo internacional en los Territorios Palestinos (Seitz, 2003, p. 50). El ISM ha logrado tener varios equipos desplegados simultáneamente en Cisjordania y Gaza con el continuo reclutamiento de activistas internacionales, y con ellos apoya las manifestaciones que se producen semanalmente para protestar contra el muro y la confiscación ilegal de tierras, así como otras acciones directas de resistencia civil contra la ocupación organizadas por la sociedad civil palestina, mientras sus activistas actúan como observadores documentando el respeto de los derechos humanos y del Derecho Internacional Humanitario, y realizan tareas de cabildeo internacional al servicio de sus socios locales (Dudouet, 2009).

10 Hebrón está situado a 30 kms al sur de Jerusalén y en ella viven alrededor de 165.000 palestinos, así como también 500 colonos israelíes concentrados en la Ciudad Vieja de Hebrón. El "Protocolo de Hebrón" de los acuerdos de Oslo de 1995 dividió la ciudad en dos zonas diferentes, H1 y H2. Así, desde 1997 la zona H1 es administrada por la AP y contiene una población de 120.000 palestinos. En la zona H2, que incluye la mayor parte de la Ciudad Vieja, el Ejército israelí restringe el movimiento de más de 30.000 palestinos, mientras permite moverse libremente a los 500 colonos israelíes.

11 Declaración recogida en la página web del ISM, http://palsolidarity.org/about/ 
Eccumenical Accompniment Programme in Palestine and Israel lleva también más de diez años apoyando a palestinos e israelíes para la transformación del conflicto y el fin de la ocupación. EAPPI fue lanzado inicialmente como parte de una campaña del Consejo Mundial de Iglesias (CMI), la principal organización ecuménica cristiana internacional, para acabar con la ocupación de Palestina a partir de una petición de 13 iglesias locales de Jerusalén, realizada en junio de 2001 (EAPPI, 2005, p. 16). A partir de esa petición, el CMI decidió poner en marcha una campaña política para acabar con la ocupación basada en el desarrollo de un programa de acompañamiento que incluyese una presencia ecuménica internacional apoyada en la experiencia de CPT, en la llamada al boicot de bienes producidos en los asentamientos ilegales israelíes en los territorios palestinos ocupados y en la llamada a la participación de las iglesias que eran miembros de la organización a unirse a los actos de resistencia no-violenta de la población palestina. ${ }^{12}$ Desde su creación, EAPPI ha desplegado en la zona a más de 1.000 voluntarios y ha expandido su área de actividad en Cisjordania para incluir Jerusalén, Belén, Hebrón, las colinas del sur de Hebrón, Tulkarem y Jayyus. Su trabajo se ha centrado en la protección de comunidades vulnerables, la observación y denuncia de abusos contra los derechos humanos y en el apoyo de grupos israelíes y palestinos que actúan de manera no-violenta para acabar con la ocupación y alcanzar la paz.

International Women's Peace Service es la única de estas iniciativas cuyos equipos de paz están formados exclusivamente por mujeres. La actividad de IWPS se localiza en el área de Salfit, una zona rural cisjordana cercana a Nablus, donde existen numerosos asentamientos israelíes ilegales y donde esta organización ha estado presente de manera continuada desde $2002 .{ }^{13}$ Su trabajo es apoyar la resistencia civil palestina que se opone a los abu- sos de los derechos humanos y a la confiscación y destrucción de sus tierras y propiedades. IWPS es una organización que proporciona acompañamiento protector a comunidades rurales vulnerables ante los ataques de colonos israelíes y apoya a organizaciones de base palestinas e israelíes que luchan contra la ocupación de manera noviolenta para la transformación del conflicto como, por ejemplo, a través de las campañas contra el muro o en favor del BDS contra Israel.

A diferencia de los grupos anteriores, Operazione Colomba no es una organización transnacional; se formó en 1995 a partir de un grupo de objetores de conciencia y de voluntarios italianos que formaban parte de la Comunidad Papa Juan XXIII y que decidieron poner en marcha un servicio de voluntariado de paz para contribuir en la transformación noviolenta de conflictos mediante el despliegue de equipos de voluntarios en zonas donde lo había. El trabajo de OC en los Territorios Palestinos comenzó en mayo de 2002, cuando desplegaron un equipo de observadores en la franja de Gaza para tratar de contribuir a reducir la violencia contra los civiles durante un año. Su acción se trasladó después a Cisjordania para apoyar a los grupos de la sociedad civil palestina que estaban luchando de manera no-violenta contra la construcción del muro y, a partir de 2004 y respondiendo a una petición de acompañamiento de una comunidad local, comenzaron a llevar a cabo una presencia continua en el pueblo de Al-Tuwani, en las colinas del Sur de Hebrón. Su trabajo se centra fundamentalmente en la protección de las comunidades de esa zona de los ataques de los colonos israelíes que viven en los asentamientos cercanos y en observar el respeto de los derechos humanos por parte de las fuerzas de seguridad israelíes. Con esa intención, OC apoya la resistencia civil de las comunidades palestinas, acompaña a pastores y campesinos durante sus actividades para prevenir ataques contra

12 Resolución sobre la respuesta ecuménica al conflicto Palestino-Israelí. Adoptada por el Comité Ejecutivo del Consejo Mundial de Iglesias, Ginebra, 11-14 de septiembre de 2001.

13 Application pack for long term and short term volunteers. International Womern's Peace Serice, mayo de 2012. Disponible en http://iwps.info/files/2012/05/IWPSApplicationPackMay2012.doc 
ellos, documenta y denuncia los actos de violencia y promueve la creación de espacios de reconciliación con grupos de la sociedad civil israelí.

Finalmente, el Instituto Internacional para la Noviolencia Activa (NOVACT) busca conseguir el desarrollo de una sociedad basada en la seguridad humana, libre de cualquier tipo de violencia, utilizando estrategias no-violentas para la transformación de los conflictos y la construcción de paces justas. Derivado del Programa de Construcción de Paz y Noviolencia Activa del Centro para la Innovación Social radicado en Barcelona, España, su trabajo se ha centrado en el Norte de África y en Oriente Medio desde 2005, cuando puso en marcha un proyecto para la promoción de la no-violencia en Gaza (NOVA, 2006, p. 2). En los Territorios Palestinos $y$ en Israel, NOVACT ha tratado de fortalecer los procesos locales de resistencia civil contra la ocupación, apoyándolos mediante formación en estrategias y tácticas de lucha no-violentas, coordinando esfuerzos para conseguir financiación para sus actividades y llevando a cabo acciones de cabildeo a nivel nacional e internacional para que estos procesos locales tengan un mayor impacto y modifiquen las políticas públicas, de modo que favorezcan la mejora de la seguridad humana y la construcción de una paz justa. Junto a ello, NOVACT trata de contribuir a la protección de estos procesos de empoderamiento de la violencia de los colonos israelíes y de la represión de las fuerzas de seguridad mediante su labor de observación del respeto de los derechos humanos y la creación de sistemas de alerta temprana.

\section{¿Qué hacen las intervenciones ciudadanas en los Territorios Palestinos?}

Las intervenciones ciudadanas que suponen un apoyo internacional noviolento para la resistencia civil palestina persiguen fundamentalmente cuatro objetivos. En primer lugar, estas iniciativas pretenden reducir los niveles de violencia para proteger a la población civil. Esta protección trata de alcanzar a los defensores y defensoras de derechos humanos palestinos e israelíes que se enfren- tan a la represión de sus actividades por las fuerzas de seguridad, pero también a las comunidades y grupos más vulnerables ante el acoso y los ataques de los colonos israelíes. En segundo lugar, estas iniciativas quieren contribuir a la transformación noviolenta del conflicto y la construcción de una paz justa y duradera, para lo que creen necesario el cambio en la distribución de poder entre los actores implicados en la disputa y una apuesta por la lucha no-violenta. Por eso, en tercer lugar, estas intervenciones quieren fortalecer los procesos de empoderamiento locales que organizaciones de la sociedad civil palestina e israelí están llevando a cabo durante sus dinámicas de lucha no-violenta contra la ocupación, para ayudarles a reducir sus vulnerabilidades y facilitar la construcción de un movimiento con mayor capacidad de influencia en la evolución del conflicto. De esta manera, las intervenciones ciudadanas quieren conseguir un cuarto objetivo, que es la extensión de la cultura de paz y la noviolencia en las sociedades palestina e israelí para romper el largo ciclo de violencia que sacude la región.

Para lograr estos objetivos, como ya hemos dejado ver en los anteriores apartados, las intervenciones internacionales noviolentas utilizan de manera simultánea cuatro tipos de estrategias diferentes. La estrategia que tiene una mayor visibilidad es la presencia física sobre el terreno, acompañando a las organizaciones locales o a las comunidades vulnerables. Esta presencia sirve como elemento disuasorio de la violencia y como mecanismo fundamental para la recolección de información de primera mano y poder dar testimonio, lo que forma parte de la segunda estrategia de acción de estas intervenciones, la de información y comunicación. Con esa información recogida de primera mano, desarrollan una estrategia de diplomacia civil que les lleva a actuar como sistemas de alerta temprana ante el deterioro de la situación en el terreno o a contactar con autoridades y representantes de la sociedad civil locales, nacionales e internacionales para llevar a cabo labores de cabildeo que les permitan transmitir sus preocupaciones y atraer la atención hacia las problemáticas de sus socios locales. Además, estas intervenciones ciu- 
dadanas que están actuando en los Territorios Palestinos y en Israel llevan a cabo una estrategia de apoyo a los grupos y comunidades con las que trabajan en el terreno. Este apoyo tiene un claro carácter técnico en forma de programas de formación y de cooperación en aquellas áreas donde los socios locales tienen carencias (estrategia y dinámicas de la acción no-violenta, autoprotección, comunicación, informática, captación de recursos, etc.), y un importante componente moral al ser la expresión de la solidaridad de la sociedad civil internacional que les muestra que no están solos.

Para generar la protección de los grupos y comunidades vulnerables y para facilitar el empoderamiento de las organizaciones locales que permita la transformación del conflicto, las estrategias de las intervenciones ciudadanas buscan aumentar los costos de la violencia y la represión. Así, cuando existe la presencia de activistas internacionales y hay trabajo de diplomacia civil de sus entidades dándoles cobertura en las instancias locales, nacionales e internacionales, la violencia se hace menos probable porque su utilización implica un mayor coste y los victimarios se inhiben o la practican a un nivel más moderado. De esta manera, se produce un ejercicio de disuasión de la violencia y se amplía el espacio disponible para la acción política, creando escenarios donde la resistencia civil contra la ocupación puede crecer y organizarse de manera autónoma. Paralelamente, las intervenciones ciudadanas también tratan de aumentar la visibilidad de las acciones de los socios locales a nivel nacional e internacional, situándolas en su contexto y alejándolas de prejuicios y descalificaciones interesadas, para mostrar la legitimidad de su lucha no-violenta en términos de defensa de los derechos humanos y de respeto del derecho internacional.

\section{Resultados alcanzados por las intervenciones ciudadanas y desafíos pendientes}

Nuestra investigación ha comprobado que estas intervenciones internacionales ciudadanas han conseguido resultados en sus objetivos de reducir la violencia, fortalecer los procesos de empoderamiento social de grupos vulnerables y en la extensión de la cultura de paz y la noviolencia. Sin embargo, debemos apuntar que estos resultados son limitados hasta el momento y que su contribución a la transformación del conflicto no parece ser muy significativa hasta ahora, aunque hay señales que indican un posible cambio en las dinámicas de algunos de los actores en disputa, especialmente en el lado palestino.

En el caso de la prevención de la violencia, las intervenciones ciudadanas han conseguido una reducción de la misma en distintos escenarios. Por ejemplo, ISM señala que desde que se producen estas intervenciones internacionales ciudadanas la utilización de munición real contra los manifestantes por parte de las fuerzas de seguridad israelíes se ha reducido y se ha prohibido su utilización. Parece también evidente que la presencia de equipos de CPT u otras organizaciones israelíes e internacionales en los controles de seguridad reduce el acoso de la policía o el ejército contra los palestinos jóvenes y adultos, y facilita su tránsito por dichos controles, como sucede en el caso de los de la Ciudad Vieja de Hebrón. La presencia de activistas internacionales también ha disminuido los ataques que sufren pequeñas comunidades rurales palestinas por parte de los colonos israelíes, como el caso de EAPPI en Yanoun.

En el caso del fortalecimiento de los procesos locales de empoderamiento social, parece evidente que el apoyo de las intervenciones internacionales noviolentas ha estimulado el crecimiento de las organizaciones, ha aumentado su impacto en la opinión pública nacional e internacional, ha facilitado la agencia local sobre sus objetivos y estrategias, y ha fomentado la creación de redes y espacios de coordinación y colaboración no solamente entre ellos, sino también con las organizaciones que realizan estas iniciativas ciudadanas y con grupos de la sociedad civil israelí. Fruto de este empoderamiento ha sido la campaña puesta en marcha para evitar la expulsión de las poblaciones palestinas de las colinas 
del sur de Hebrón, en la zona de Masafer Yatta, ${ }^{14}$ que ha conseguido frenarla hasta ahora, o acciones de movilización a mayor escala como el campamento de Bab al Shams a comienzos de 2013, para reivindicar el derecho a la tierra de los palestinos y protestar contra la política israelí de construcción de asentamientos en Jerusalén Este. ${ }^{15}$ De esta manera, podemos afirmar que desde el inicio de la segunda intifada, el movimiento de resistencia civil en los Territorios Palestinos se ha ampliado con el apoyo de las intervenciones internacionales ciudadanas y ha alcanzado una mayor influencia en el ámbito de las políticas públicas desarrolladas por Israel y la Autoridad Palestina, pero también en el de organismos internacionales como la Unión Europea. ${ }^{16}$

Las intervenciones internacionales ciudadanas también están consiguiendo extender la cultura de paz y la noviolencia en las sociedades palestina e israelí, fomentando la construcción de amplios movimientos no-violentos que superen la fragmentación de la vida política palestina y que fomenten la cooperación con grupos israelíes que persiguen el fin de la ocupación y alcanzar una paz justa. Un claro ejemplo de esta cooperación es la extensión de los comités populares que organizan la resistencia civil no-violenta a nivel local y que están abiertos a todos los activistas independientemente de su tendencia o afiliación política, que colaboran con organizaciones israelíes y el surgimiento del Comité de Coordinación de la Lucha Popular. Poco a poco, estas iniciativas ciudadanas están contribuyendo a crear una masa crítica favorable a la lucha noviolenta que aprende sus métodos, conoce sus dinámicas y está dispuesta a superar las fracturas de la sociedad palestina para poner punto y final a la ocupación. Además, estos movimientos noviolentos están discutiendo las narrativas dominantes que han glorificado la lucha armada a lo largo de la historia del conflicto y están facilitando el cambio de paradigma de la cultura de la violencia a la cultura de paz.

Por otro lado, estas intervenciones han cosechado un escaso éxito en su objetivo de transformar el conflicto ya que, si bien es cierto que su trabajo contribuye a fortalecer al movimiento de resistencia civil palestino y a aumentar el perfil de su lucha y sus reivindicaciones ante la opinión pública y las instituciones internacionales, su capacidad para fomentar el cambio de las políticas públicas de Israel hacia los Territorios Palestinos es muy limitada. Esto se debe a que Israel sigue siendo capaz de asumir los costes políticos que conlleva la ocupación, pero también a que estas intervenciones no han sabido generar una influencia significativa sobre la sociedad israelí para que deje de tolerar el status quo y demande cambios a sus gobernantes. Es en el lado palestino en el que parece existir una dinámica positiva en favor de la transformación noviolenta del conflicto, dado que amplios sectores de la sociedad y la mayoría de las facciones políticas parecen haberle dado una oportunidad a la resistencia civil, dándole prioridad a la lucha no-violenta sobre la lucha armada. ${ }^{17}$

14 La organización de derechos humanos B'tselem ofrece un detallado análisis de este proceso en http://www.btselem.org/ south_hebron_hills/firing_zone_918

15 Descripciones de esta acción, del contexto donde se produjo y de las repercusiones que tuvo pueden encontrarse en el artículo: "One, two, a hundred Bab al-Shams - new facts on the ground in Palestine?", Waging Nonviolence, 24 de enero de 2013. Disponible en http://wagingnonviolence.org/feature/one-two-a-hundred-bab-al-shams-new-facts-on-the-ground-in-palestine/

16 Una muestra de esto último es la directiva europea que pretende incluir en cualquier acuerdo que firme la Unión Europea con Israel una cláusula que explícitamente excluya de los mismos a las colonias israelíes en Cisjordania. "EU takes tougher stance on Israeli settlements", The Guardian, 16 de julio de 2013. Disponible en http://www.theguardian.com/world/2013/jul/16/ eu-israel-settlement-exclusion-clause

17 Esta percepción está respaldada por la evolución de las encuestas de opinión en los Territorios Palestinos que evidencian un rechazo cada vez mayor a la utilización de la lucha armada y un amplio apoyo a la resistencia noviolenta que alcanza en la actualidad al 80\% de la población. "Poll: Hamas loses popularity among Palestinians", The Jerusalem Post, 4 de Octubre de 2013. Disponible en http://www.jpost.com/Middle-East/Poll-Hamas-continues-to-lose-popularity-among-Palestinians-309372 
Sin embargo, tal y como hemos mencionado anteriormente, aunque podemos otorgar una gran importancia a muchos de estos resultados, hay que reconocer que todavía se muestran bastante limitados en el contexto general del conflicto. $\mathrm{Y}$ es que la violencia continúa presente de manera directa en la vida de los palestinos cuando, por ejemplo, los manifestantes que protestan pacíficamente contra el muro son dispersados mediante la utilización de medios violentos, aunque supuestamente no letales como pelotas de goma, granadas de gases lacrimógenos y bombas de sonido, o cuando las poblaciones sufren castigos colectivos y se restringe su movimiento. También sufren la violencia de manera indirecta en numerosas ocasiones, como en el caso del mantenimiento de un sistema de justicia que facilita la impunidad de los que cometen violaciones de derechos humanos (Amnesty International, 2013). Además, los procesos de empoderamiento social que estas iniciativas ciudadanas están fortaleciendo presentan varias incógnitas respecto a su sostenibilidad, no solamente en torno a su capacidad para movilizar recursos sino también sobre su capacidad para consolidar esa cooperación por encima de las fracturas políticas e ideológicas y poder desarrollar estrategias de acción en torno a objetivos compartidos. El papel de las intervenciones internacionales noviolentas puede ser muy importante para facilitar esa consolidación. Por último, también hay que contemplar la posibilidad de que las intervenciones internacionales noviolentas pueden correr el riesgo de que se fracase en la extensión de la cultura de paz si la resistencia civil no consigue avanzar en la transformación del conflicto y en la construcción de una paz más justa. Esto se debe a que la falta de avances significativos en la protección de los derechos humanos y el respeto del derecho internacional en los Territorios Palestinos puede hacer dudar de la viabilidad de la acción no-violenta como método de lucha para poner fin a la ocupación, y la frustración de la sociedad palestina puede conducir de nuevo a una apuesta por la resistencia armada. Todas estas limitaciones que aparecen en los resultados del trabajo de las intervenciones internacionales ciudadanas hasta la fecha son los próximos retos y desafíos que tienen que abordar.

\section{Conclusiones}

Este análisis del fenómeno de las intervenciones internacionales ciudadanas en los Territorios Ocupados nos permite extraer varias conclusiones. En primer lugar, se evidencia cómo la presencia de activistas internacionales en Oriente Medio ya no es solamente para mostrar su solidaridad con la población palestina o contribuir a los esfuerzos humanitarios y de cooperación para el desarrollo, sino que destaca como parte de la sociedad civil global se ha implicado en iniciativas ciudadanas para la prevención de la violencia, la transformación noviolenta de los conflictos y la construcción de una paz más justa y duradera en la región.

En segundo lugar, hay que señalar que más allá de la retórica de la violencia que existe alrededor de la resistencia palestina contra la ocupación y cuyo amplio respaldo en la población no se puede negar, en los Territorios Palestinos existen consistentes movimientos de base que abogan por la transformación del conflicto de manera noviolenta. Si bien es cierto que en la historia del movimiento nacional palestino la resistencia civil tiene un papel mayoritariamente subordinado a la lucha armada, en los últimos años parece haberse abierto una ventana de oportunidad donde está predominando la lucha no-violenta como estrategia de resistencia y a la que se están sumando la mayoría de los actores políticos y sociales del movimiento nacional palestino. En el contexto de Oriente Medio esto puede suponer un ejemplo de la viabilidad de la lucha no-violenta para la transformación de los conflictos de la zona que estimule la extensión de movimientos de resistencia civil en otros países al calor de las Primaveras Árabes.

En tercer lugar, debemos resaltar la importancia de las contribuciones de las intervenciones internacionales ciudadanas para la lucha contra la ocupación de los Territorios Palestinos. El apoyo internacional noviolento está claramente aumentando la protección y el empoderamiento de esos 
movimientos de base que practican una resistencia civil en medio de la represión de las fuerzas ocupantes; también está desafiando el militarismo y la cultura de la violencia que se encuentra instalada profundamente en la mentalidad de la sociedad para facilitar un cambio a favor de la extensión de la cultura de la paz y la noviolencia. Es sintomático de este proceso la construcción social de una nueva imagen de héroe, que ahora incluye también a los resistentes que llevan a cabo una lucha sin armas contra la ocupación. Además, estas intervenciones de la sociedad civil global están probando una vez más que es posible la colaboración de grupos "occidentales" con movimientos y organizaciones árabes y musulmanes, superando sus diferencias culturales para trabajar en la construcción de la paz de acuerdo a los intereses y necesidades locales.

\section{Referencias}

Addameer. (2012). Palestinian political prisoners in Israeli prisons. Jerusalem: Addameer.

Amnesty International. (2013). Annual Report 2013 - Israel and occupied Palestinian territories. The state of the world's buman rights. Recuperado de http://www.amnesty.org/en/region/ israel-and-occupied-palestinian-territories / report-2013

Barghouti, O. (2006). Putting Palestine back on the map: Boycott as Civil Resistance. Journal of Palestine Studies, 35(3), 51-57.

Broning, M. (2011). The Politics of change in Palestine. State-building and non-violent resistance. New York: Pluto Press.

Cortright, D. (2008). Peace: A bistory of movements and ideas. Cambridge: Cambridge University Press.
Finalmente, en cuarto lugar, tenemos que reconocer que aún no es posible evaluar la contribución de las intervenciones internacionales ciudadanas a la transformación del conflicto. Nuestros primeros hallazgos indican que su trabajo no ha supuesto cambios significativos en la distribución de la estructura de poder en el mismo, aunque parece que la oportunidad que los distintos actores políticos palestinos le están dando a la resistencia civil en los últimos años está, en parte, propiciada por los resultados que han conseguido las organizaciones locales, practicando este modelo de lucha con el apoyo de las intervenciones internacionales noviolentas. Sin embargo, para confirmar este punto será preciso continuar esta investigación e incluir en el análisis al conjunto de los actores políticos palestinos.

Checa Hidalgo, D. (2011). Intervenciones internacionales noviolentas. Herramientas para la transformación de conflictos. Convergencia, 18(56), 87-109.

(2012). Origen y desarrollo de las intervenciones internacionales no violentas para la transformación de conflictos. Historia Actual Online, 28, 21-35.

Chenoweth, E., \& Stephan, M. J. (2011). Why civil resistance works? The strategic logic of nonviolent conflict. New York: Columbia University Press.

De Jong, A. (2012). The Gaza Freedom Flotilla: Human Rights, Activism and Academic Neutrality. Social Movement Studies, 11(2), 193-209.

Dudouet, V. (2009). Cross-border non-violent advocacy during the second Palestinian intifada: The 
case of International Solidarity Movement. En H. Clark (Ed.), People Power. Unarmed resistance and global solidarity (pp. 125-138)). London/New York: Pluto Press.

EAPPI. (2005). Theological reflection on accompaniment. Ecumenical Accompaniment Programme in Palestine and Israel. Ginebra: World Council of Churches. Recuperado de http://eappi.org/ en/resources/eappi-publications.html?no_ca che $=1 \& \operatorname{cid}=40360 \& \operatorname{did}=23696 \& \operatorname{sechash}=a$ $7 \mathrm{ca} 7 \mathrm{c} 43$

Galtung, J. (1969). Violence, peace, and peace research. Journal of peace research, 6 (3), 167-191.

James, W. (agosto, 1910). The Moral Equivalent of War. McClure's Magazine, 463-468. Recuperado de http://www.constitution.org/wj/meow.htm

Kayyali, A. S. (1970). The Palestinian Arab reactions to Zionism and the British Mandate 19171939. (Tesis doctoral inedita). University of London, Londres, Reino Unido.

Kern, K. (2000). From Haiti to Hebron with a Brief Stop in Washington, D.C. The CPT Experiment. En C. Sampson, \& J. P. Lederach (Eds.), From The Ground Up. Mennonite Contributions to International Peacebuilding (pp. 183-200). Oxford: Oxford University Press.

King, M. E. (2007). A Quiet Revolution: The First Palestinian Intifada and Nonviolent Resistance. New York: Nation Books.

Lederach, J. P. (1997). Building Peace: Sustainable Reconciliation In Divided Societies. Washington: United States Institute of Peace.

Moser-Puangsuwan, Y. (2000). From the peace army to Sipaz: A chronology of grassroots initiatives in unarmed peacekeeping. En Y. MoserPuangsuwan\& T. Weber (Eds.), Nonviolent intervention across borders. A recurrent vision (pp. 341-356). Honolulu: Spark M. Matsunaga Institute for Peace/University of Hawaii.
Norman, J.M. (2010). The Second Palestinian Intifada. Civil Resistance. Londres/New York: Routledge.

NOVA. (2006). La resistencia noviolenta a l'Orient Mitja. Quaderns NoVA, 1. Cerdanyola del Valles: NOVA.

Pappe, I. (2004). A History of Modern Palestine: One Land. Two Peoples. Cambridge: Cambridge University Press.

Pappé, I. (2006). The 1948 Ethnic Cleansing of Palestine. Journal of Palestine Studies, 36(1), 6-20.

Qumsiyeh, M. B. (2011). Popular resistance in Palestine: A bistory of hope and empowerment. New York: Pluto Press.

Seitz, Ch. (2003). ISM at the Crossroads: the evolution of the International Solidarity Movement. Journal of Palestine Studies, 32(4), 50-67.

. (2011). Tracking Palestinian public support for armed resistance during the peace process and its demise. Ramallah: Jerusalem Media and Communications Centre.

Sharp, G. (1989). The Intifadah and Nonviolent Struggle. Journal of Palestine Studies, 19(1), 3-13.

Rigby, A. (1995). Unofficial Nonviolent Intervention: Examples from the IsraeliPalestinian conflict. Journal of Peace Research, 32(4), 453-467. . (2010). Palestinian Resistance and Nonviolence. Jerusalem: Passia.

Weber, T. (1993). From Maude Royden's Peace Army to the Gulf Peace Team: An assessment of unarmed interpositionary peace forces. Journal of Peace Research, 30(1), 45-64.

. (1996). Gandhi's Peace Army: The Shanti Sena and Unarmed Peacekeeping. New York: Syracuse University Press. 